\title{
A Criterion for Subfamilies of Multivalent Functions of Reciprocal Order with Respect to Symmetric Points
}

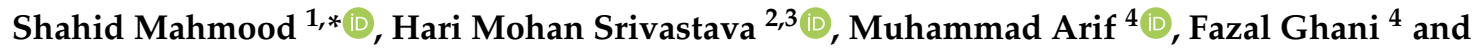 \\ Eman S. A. AbuJarad ${ }^{5}$ \\ 1 Department of Mechanical Engineering, Sarhad University of Science and Information Technology, \\ Peshawar 25000, Pakistan \\ 2 Department of Mathematics and Statistics, University of Victoria, Victoria, BC V8W 3R4, Canada; \\ harimsri@math.uvic.ca \\ 3 Department of Medical Research, China Medical University Hospital, China Medical University, \\ Taichung 40402, Taiwan \\ 4 Department of Mathematics, Abdul Wali Khan University Mardan, Mardan 23200, Pakistan; \\ marifmaths@awkum.edu.pk (M.A.); fazalghanimaths@gmail.com (F.G.) \\ 5 Department of Mathematics, Aligarh Muslim University, Aligarh 202002, India; emanjarad2@gmail.com \\ * Correspondence: shahidmahmood757@gmail.com
}

Received: 19 May 2019; Accepted: 20 June 2019; Published: 25 June 2019

Abstract: In the present research paper, our aim is to introduce a new subfamily of $p$-valent (multivalent) functions of reciprocal order. We investigate sufficiency criterion for such defined family.

Keywords: multivalent functions; starlike functions; close-to-convex functions

MSC: Primary 30C45, 30C10; Secondary 47B38

\section{Introduction}

Let us suppose that $\mathcal{A}_{p}$ represents the class of $p$-valent functions $f(z)$ that are holomorphic (analytic) in the region $\mathbb{E}=\{z:|z|<1\}$ and has the following Taylor series representation:

$$
f(z)=z^{p}+\sum_{k=1}^{\infty} a_{p+k} z^{p+k}
$$

Two points $p$ and $p^{\prime}$ are said to be symmetrical with respect to $o$ if $o$ is the midpoint of the line segment $p p^{\prime}$.

If $f(z)$ and $g(z)$ are analytic in $\mathcal{E}$, we say that $f(z)$ is subordinate to $g(z)$, written as $f(z) \prec g(z)$, if there exists a Schwarz function, $w(z)$, which is analytic in $\mathcal{E}$ with $w(0)=0$ and $|w(z)|<1$ such that $f(z)=g(w(z))$. Furthermore, if the function $g(z)$ is univalent in $\mathcal{E}$, then we have the following equivalence, see [1].

$$
f(z) \prec g(z)(z \in \mathcal{E}) \Longleftrightarrow f(0)=g(0) \quad \text { and } \quad f(\mathcal{E}) \subset g(\mathcal{E})
$$

Let $\mathcal{N}_{\alpha}$ denotes the class of starlike functions of reciprocal order $\alpha(\alpha>1)$ and is given below

$$
\mathcal{N}_{\alpha}:=\left\{f(z) \in \mathcal{A}: \operatorname{Re}\left(\frac{z f^{\prime}(z)}{f(z)}\right)<\alpha,(z \in \mathbb{E})\right\} .
$$


This class was introduced by Uralegaddi et al. [2] amd further studied by the Owa et al. [3]. After that Nunokawa and his coauthors [4] proved that $f(z) \in \mathcal{N}_{\alpha}, 0<\alpha<\frac{1}{2}$, if and only if the following inequality holds

$$
\left|\frac{2 \alpha z f^{\prime}(z)}{f(z)}-1\right|<1,(z \in \mathbb{E}) .
$$

Later on, Owa and Srivastava [5] in 2002 generalized this idea for the classes of multivalent convex and starlike functions of reciprocal order $\alpha(\alpha>p)$, and further studied by Polatoglu et al. [6]. For more details of the related concepts, see the article of Dixit et al. [7], Uyanik et al. [8], and Arif et al. [9].

For $-1 \leq t<s \leq 1$ with $s \neq 0 \neq t, 0<\alpha<1$, and $p \in \mathbb{N}$, we introduce a subclass of $\mathcal{A}_{p}$ consisting of all analytic $p$-valent functions of reciprocal order $\alpha$, denoted by $\mathcal{N}_{\alpha}^{p} \mathcal{S}(s, t)$ and is defined as

$$
\mathcal{N}_{\alpha}^{p} \mathcal{S}(s, t)=\left\{f(z) \in \mathcal{A}_{p}: \operatorname{Re}\left(\frac{\left(s^{p}-t^{p}\right) z f^{\prime}(z)}{f(s z)-f(t z)}\right)<\frac{p}{\alpha},(z \in \mathbb{E})\right\},
$$

or equivalently

$$
\left|\frac{\left(s^{p}-t^{p}\right) z f^{\prime}(z)}{f(s z)-f(t z)}-\frac{p}{2 \alpha}\right| \leq \frac{p}{2 \alpha} .
$$

Many authors studied sufficiency conditions for various subclasses of analytic and multivalent functions, for details see [4,10-17].

We will need the following lemmas for our work.

Lemma 1 (Jack's lemma [18]). Let $\Psi$ be a non-constant holomorphic function in $\mathbb{E}$ and if the value of $|\Psi|$ is maximum on the circle $|z|=r<1$ at $z_{\circ}$, then $z_{\circ} \Psi^{\prime}\left(z_{\circ}\right)=k \Psi\left(z_{\circ}\right)$, where $k \geq 1$ is a real number.

Lemma 2 (See [1]). Let $\mathfrak{H} \subset \mathbb{C}$ and let $\Phi: \mathbb{C}^{2} \times \mathbb{E}^{*} \rightarrow \mathbb{C}$ be a mapping satisfying $\Phi(i a, b, z) \notin \mathfrak{H}$ for $a, b \in \mathbb{R}$ such that $b \leq-\frac{1+a^{2}}{2}$. If $p(z)=1+c_{1} z^{1}+c_{2} z^{2}+\cdots$ is regular in $\mathbb{E}^{*}$ and $\Phi\left(p(z), z p^{\prime}(z), z\right) \in \mathfrak{H}$ $\forall z \in \mathbb{E}^{*}$, then $\operatorname{Re}(p(z))>0$.

Lemma 3 (See [15]). Let $p(z)=1+c_{1} z+c_{2} z^{2}+\cdots$ be analytic in $\mathbb{E}$ and $\eta$ be analytic and starlike (with respect to the origin) univalent in $\mathbb{E}$ with $\eta(0)=0$. If $z p^{\prime}(z) \prec \eta(z)$, then

$$
p(z) \prec 1+\int_{0}^{z} \frac{\eta(t)}{t} d t .
$$

This result is the best possible.

\section{Main Results}

Theorem 1. Let $f(z) \in \mathcal{A}_{p}$ and satisfies

$$
\sum_{n=1}^{\infty}\left(\alpha(p+n)+p \frac{\left(s^{p+n}-t^{p+n}\right)}{\left(s^{p}-t^{p}\right)}\right)\left|a_{n+p}\right| \leq \frac{p}{2}(1-|2 \alpha-1|) .
$$

Then $f(z) \in \mathcal{N}_{\alpha}^{p} \mathcal{S}(s, t)$.

Proof. Let us assume that the inequality (5) holds. It suffices to show that

$$
\left|\frac{2 \alpha\left(s^{p}-t^{p}\right) z f^{\prime}(z)}{f(s z)-f(t z)}-p\right| \leq p .
$$


Consider

$$
\begin{aligned}
& \left|\frac{2 \alpha\left(s^{p}-t^{p}\right) z f^{\prime}(z)}{f(s z)-f(t z)}-p\right| \\
= & \left|\frac{p(2 \alpha-1)\left(s^{p}-t^{p}\right) z^{p}+\sum_{n=1}^{\infty}\left(2 \alpha(p+n)\left(s^{p}-t^{p}\right)-p\left(s^{n+p}-t^{n+p}\right)\right) a_{n+p} z^{n+p}}{\left(s^{p}-t^{p}\right) z^{p}+\sum_{n=1}^{\infty}\left(s^{n+p}-t^{n+p}\right) a_{n+p} z^{n+p}}\right| \\
\leq & \frac{p|2 \alpha-1|\left(s^{p}-t^{p}\right)+\sum_{n=1}^{\infty}\left(2 \alpha(p+n)\left(s^{p}-t^{p}\right)+p\left(s^{n+p}-t^{n+p}\right)\right)\left|a_{n+p}\right|}{\left(s^{p}-t^{p}\right)-\sum_{n=1}^{\infty}\left(s^{n+p}-t^{n+p}\right)\left|a_{n+p}\right|}
\end{aligned}
$$

The last expression is bounded above by $p$ if

$$
\begin{aligned}
& p|2 \alpha-1|\left(s^{p}-t^{p}\right)+\sum_{n=1}^{\infty}\left(2 \alpha(p+n)\left(s^{p}-t^{p}\right)+p\left(s^{n+p}-t^{n+p}\right)\right)\left|a_{n+p}\right| \\
< & p\left\{\left(s^{p}-t^{p}\right)-\sum_{n=1}^{\infty}\left(s^{n+p}-t^{n+p}\right)\left|a_{n+p}\right|\right\} .
\end{aligned}
$$

Hence

$$
\sum_{n=1}^{\infty}\left(\alpha(p+n)+p \frac{\left(s^{p+n}-t^{p+n}\right)}{\left(s^{p}-t^{p}\right)}\right)\left|a_{n+p}\right| \leq \frac{p}{2}(1-|2 \alpha-1|) .
$$

This shows that $f(z) \in \mathcal{N} \mathcal{S}_{p}(s, t, \alpha)$. This completes the proof.

Theorem 2. If $f(z) \in \mathcal{A}_{p}$ satisfies the condition

$$
\left|1+\frac{z f^{\prime \prime}(z)}{f^{\prime}(z)}-\frac{z(f(s z)-f(t z))^{\prime}}{f(s z)-f(t z)}\right|<1-\alpha, \quad\left(\frac{1}{2} \leq \alpha<1\right)
$$

then $f(z) \in \mathcal{N}_{\alpha}^{p} \mathcal{S}(s, t)$.

Proof. Let us set

$$
q(z)=\frac{1-\frac{\alpha\left(s^{p}-t^{p}\right) z f^{\prime}(z)}{p(f(s z)-f(t z))}}{1-\alpha}-1 .
$$

Then clearly $q(z)$ is analytic in $\mathbb{E}$ with $q(0)=0$. Differentiating logarithmically, we have

$$
1+\frac{z f^{\prime \prime}(z)}{f^{\prime}(z)}-\frac{z(f(s z)-f(t z))^{\prime}}{f(s z)-f(t z)}=-\frac{(1-\alpha) z q^{\prime}(z)}{(\alpha-(1-\alpha) q(z))} .
$$

So

$$
\left|1+\frac{z f^{\prime \prime}(z)}{f^{\prime}(z)}-\frac{z(f(s z)-f(t z))^{\prime}}{f(s z)-f(t z)}\right|=\left|-\frac{(1-\alpha) z q^{\prime}(z)}{(\alpha-(1-\alpha) q(z))}\right| .
$$

From (7), we have

$$
\left|\frac{(1-\alpha) z q^{\prime}(z)}{(\alpha-(1-\alpha) q(z))}\right|<1-\alpha
$$

Next, we claim that $|q(z)|<1$. Indeed, if not, then for some $z_{\circ} \in \mathbb{E}$, we have

$$
\max _{|z| \leq\left|z_{0}\right|}|q(z)|=\left|q\left(z_{0}\right)\right|=1
$$


Applying Jack's lemma to $q(z)$ at the point $z_{0}$, we have

$$
q\left(z_{0}\right)=e^{i \theta}, \frac{z_{0} q^{\prime}\left(z_{0}\right)}{q\left(z_{0}\right)}=k, k \geq 1
$$

Then

$$
\begin{aligned}
\left|1+\frac{z_{0} f^{\prime \prime}\left(z_{0}\right)}{f^{\prime}\left(z_{0}\right)}-\frac{z\left(f\left(s z_{0}\right)-f\left(t z_{0}\right)\right)^{\prime}}{f\left(s z_{0}\right)-f\left(t z_{0}\right)}\right| & =\left|\frac{(1-\alpha) z_{0} q^{\prime}\left(z_{0}\right)}{\left(\alpha-(1-\alpha) q\left(z_{0}\right)\right)}\right| \\
& =|1-\alpha| \frac{z_{0} q^{\prime}\left(z_{0}\right)}{q\left(z_{0}\right)}\left(\frac{1}{(1-\alpha)-\alpha e^{-i \theta}}\right) \mid \\
& =|1-\alpha|\left|\frac{k}{\alpha e^{-i \theta}-(1-\alpha)}\right| \\
& \geq|1-\alpha|\left|\frac{1}{(1-\alpha)-\alpha e^{-i \theta}}\right| .
\end{aligned}
$$

Therefore

$$
\left|1+\frac{z_{0} f^{\prime \prime}\left(z_{0}\right)}{f^{\prime}\left(z_{0}\right)}-\frac{z\left(f\left(s z_{0}\right)-f\left(t z_{0}\right)\right)^{\prime}}{f\left(s z_{0}\right)-f\left(t z_{0}\right)}\right|^{2} \geq \frac{(1-\alpha)^{2}}{(1-\alpha)^{2}+\alpha^{2}-2 \alpha(1-\alpha) \cos \theta} .
$$

Now the right hand side has minimum value at $\cos \theta=-1$, therefore we have

$$
\left|1+\frac{z_{0} f^{\prime \prime}\left(z_{0}\right)}{f^{\prime}\left(z_{0}\right)}-\frac{z\left(f\left(s z_{0}\right)-f\left(t z_{0}\right)\right)^{\prime}}{f\left(s z_{0}\right)-f\left(t z_{0}\right)}\right|^{2} \geq(1-\alpha)^{2} .
$$

But this contradicts (7). Hence we conclude that $|q(z)|<1$ for all $z \in \mathbb{E}$, which shows that

$$
\left|\frac{1-\frac{\alpha\left(s^{p}-t^{p}\right) z f^{\prime}(z)}{p(f(s z)-f(t z))}}{1-\alpha}-1\right|<1
$$

This implies that

$$
\left|\frac{\left(s^{p}-t^{p}\right) z f^{\prime}(z)}{p(f(s z)-f(t z))}-1\right|<\frac{1}{\alpha}-1
$$

Now we have

$$
\begin{aligned}
\left|\frac{\left(s^{p}-t^{p}\right) z f^{\prime}(z)}{p(f(s z)-f(t z))}-\frac{1}{2 \alpha}\right| & \leq\left|\frac{\left(s^{p}-t^{p}\right) z f^{\prime}(z)}{p(f(s z)-f(t z))}-1\right|+\left|1-\frac{1}{2 \alpha}\right| \\
& <\frac{1}{\alpha}-1+1-\frac{1}{2 \alpha} \\
& =\frac{1}{2 \alpha} .
\end{aligned}
$$

This implies that $f(z) \in \mathcal{N}_{\alpha}^{p} \mathcal{S}(s, t)$.

Theorem 3. If $f(z) \in \mathcal{A}_{p}$ satisfies the condition

$$
\operatorname{Re}\left(-1-\frac{z f^{\prime \prime}(z)}{f^{\prime}(z)}+\frac{z(f(s z)-f(t z))^{\prime}}{f(s z)-f(t z)}\right)>\left\{\begin{array}{cc}
\frac{\alpha}{2(\alpha-1)}, & 0 \leq \alpha \leq \frac{1}{2} \\
\frac{\alpha-1}{2 \alpha}, & \frac{1}{2} \leq \alpha<1,
\end{array}\right.
$$

then $f(z) \in \mathcal{N}_{\alpha}^{p} \mathcal{S}(s, t)$ for $0 \leq \alpha<1$. 
Proof. Let

$$
q(z)=\frac{\frac{p(f(s z)-f(t z))}{\left(s^{p}-t^{p}\right) z f^{\prime}(z)}-\alpha}{1-\alpha} .
$$

Then clearly $q(z)$ is analytic in $\mathbb{E}$. Applying logarithmic differentiation, we have

$$
-1-\frac{z f^{\prime \prime}(z)}{f^{\prime}(z)}+\frac{z(f(s z)-f(t z))^{\prime}}{f(s z)-f(t z)}=\frac{(1-\alpha) z q^{\prime}(z)}{\alpha+(1-\alpha) q(z)}=\Psi\left(q(z), z q^{\prime}(z), z\right)
$$

where

$$
\Psi(u, v ; t)=\frac{(1-\alpha) v}{\alpha+(1-\alpha) u} .
$$

Now for all $x, y \in \mathbb{R}$ satisfying the inequality $y \leq-\frac{1+x^{2}}{2}$, we have

$$
\Psi(i x, y, z)=\frac{(1-\alpha) y}{\alpha+(1-\alpha) i x}
$$

Therefore

$$
\begin{aligned}
\operatorname{Re}(\Psi(i x, y, z)) & \leq-\frac{\alpha(1-\alpha)\left(1+x^{2}\right)}{2\left(\alpha^{2}+(1-\alpha)^{2} x^{2}\right)} \\
& \leq\left\{\begin{array}{cc}
\frac{\alpha}{2(\alpha-1)}, & 0 \leq \alpha \leq \frac{1}{2} \\
\frac{\alpha-1}{2 \alpha}, & \frac{1}{2} \leq \alpha<1
\end{array}\right.
\end{aligned}
$$

We set

$$
\Lambda=\left\{\zeta: \operatorname{Re}(\zeta)>\left\{\begin{array}{cl}
\frac{\alpha}{2(\alpha-1)}, & 0 \leq \alpha \leq \frac{1}{2} \\
\frac{\alpha-1}{2 \alpha}, & \frac{1}{2} \leq \alpha<1
\end{array}\right\}\right.
$$

Then $\Psi(i x, y ; z) \notin \Lambda$ for all real $x, y$ such that $y \leq-\frac{1+x^{2}}{2}$. Moreover, in view of (10), we know that $\Psi\left(q(z), z q^{\prime}(z), z\right) \in \Lambda$. So applying Lemma 2, we have

$$
\operatorname{Re}(q(z))>0
$$

which shows that the desired assertion of Theorem 3 holds.

Theorem 4. If $f(z) \in \mathcal{A}_{p}$ satisfies

$$
\operatorname{Re} \frac{f(s z)-f(t z)}{\left(s^{p}-t^{p}\right) z f^{\prime}(z)}\left(1-\beta \frac{z f^{\prime \prime}(z)}{f^{\prime}(z)}+\beta \frac{z(f(s z)-f(t z))^{\prime}}{f(s z)-f(t z)}\right)>\frac{2 \alpha+\beta(3 \alpha-1)}{2 p}
$$

then $f(z) \in \mathcal{N}_{\alpha}^{p} \mathcal{S}(s, t)$ for $0<\alpha<1$ and $\beta \geq 0$.

Proof. Let

$$
h(z)=\frac{\frac{p(f(s z)-f(t z))}{\left(s^{p}-t^{p}\right) z f^{\prime}(z)}-\alpha}{1-\alpha} .
$$

Where $h(z)$ is clearly analytic in $\mathbb{E}$ such that $h(0)=1$. We can write

$$
\frac{p(f(s z)-f(t z))}{\left(s^{p}-t^{p}\right) z f^{\prime}(z)}=\alpha+(1-\alpha) h(z) \text {. }
$$


After some simple computation, we have

$$
-\beta \frac{z f^{\prime \prime}(z)}{f^{\prime}(z)}+\beta \frac{z(f(s z)-f(t z))^{\prime}}{f(s z)-f(t z)}=\beta \frac{\alpha+(1-\alpha)\left(h(z)+z h^{\prime}(z)\right)}{\alpha+(1-\alpha) h(z)}
$$

It follows from (12) that

$$
\begin{aligned}
& \frac{p(f(s z)-f(t z))}{\left(s^{p}-t^{p}\right) z f^{\prime}(z)}\left(1-\beta \frac{z f^{\prime \prime}(z)}{f^{\prime}(z)}+\beta \frac{z(f(s z)-f(t z))^{\prime}}{f(s z)-f(t z)}\right) \\
= & \beta(1-\alpha) z h^{\prime}(z)+(1-\alpha)(1+\beta) h(z)+\alpha(1+\beta) \\
= & \Psi\left(h(z), z h^{\prime}(z), z\right)
\end{aligned}
$$

where

$$
\Psi(u, v, t)=\beta(1-\alpha) v+(1-\alpha)(1+\beta) u+\alpha(1+\beta) .
$$

Now for some real numbers $x$ and $y$ satisfying $y \leq-\frac{1+x^{2}}{2}$, we have

$$
\begin{aligned}
\operatorname{Re}(\Psi(i x, y, z)) & \leq-\beta(1-\alpha) \frac{1+x^{2}}{2}+\alpha(1+\beta) \\
& =\frac{1}{2}(2 \alpha+\beta(3 \alpha-1)) .
\end{aligned}
$$

If we set

$$
\Lambda=\left\{\zeta: \operatorname{Re}(\zeta)>\frac{1}{2}(2 \alpha+\beta(3 \alpha-1))\right\},
$$

then $\Psi(i x, y, z) \notin \Lambda$ Furthermore, by virtue of (11), we know that $\Psi\left(h(z), z h^{\prime}(z), z\right) \in \Lambda$. Thus by using Lemma 2, we have

$$
\operatorname{Re}(h(z))>0,
$$

which implies that the assertion of Theorem 4 holds true.

Theorem 5. If $f(z) \in \mathcal{A}_{p}$ satisfies the condition

$$
\left|\left(p-\frac{2 \alpha\left(s^{p}-t^{p}\right) z f^{\prime}(z)}{(f(s z)-f(t z))}\right)^{\prime}\right| \leq p \beta|z|^{\gamma},
$$

then $f(z) \in \mathcal{N}_{\alpha}^{p} \mathcal{S}(s, t)$ with $0<\alpha<1,0<\beta \leq \gamma+1$ and $\gamma \geq 0$.

Proof. Let we define

$$
\digamma(z)=z\left(p-\frac{2 \alpha\left(s^{p}-t^{p}\right) z f^{\prime}(z)}{(f(s z)-f(t z))}\right) .
$$

Then $\digamma(z)$ is regular in $\mathbb{E}$ and $\digamma(0)=0$. The condition (14) gives

$$
\left|\left(p-\frac{2 \alpha\left(s^{p}-t^{p}\right) z f^{\prime}(z)}{(f(s z)-f(t z))}\right)^{\prime}\right|=\left|\left(\frac{\digamma(z)}{z}\right)^{\prime}\right|
$$

It follows from (13) that

$$
\left|\left(\frac{\digamma(z)}{z}\right)^{\prime}\right| \leq p \beta|z|^{\gamma}
$$


This implies that

$$
\left|\left(\frac{\digamma(z)}{z}\right)\right|=\left|\int_{0}^{z}\left(\frac{\digamma(t)}{t}\right)^{\prime} d t\right| \leq \int_{0}^{z}\left|\left(\frac{\digamma(t)}{t}\right)^{\prime}\right| d t \leq \frac{p \beta|z|^{\gamma+1}}{\gamma+1},
$$

and therefore

$$
\left|\left(\frac{\digamma(z)}{z}\right)\right|<p
$$

which further gives

$$
\left|\frac{\left(s^{p}-t^{p}\right) z f^{\prime}(z)}{p(f(s z)-f(t z))}-\frac{1}{2 \alpha}\right|<\frac{1}{2 \alpha}
$$

Hence $f(z) \in \mathcal{N}_{\alpha}^{p} \mathcal{S}(s, t)$.

Theorem 6. If $f(z) \in \mathcal{A}_{p}$ satisfies

$$
\left|\frac{\left(s^{p}-t^{p}\right) z f^{\prime}(z)}{f(s z)-f(t z)}\left(1+\frac{z f^{\prime \prime}(z)}{f^{\prime}(z)}-\frac{z(f(s z)-f(t z))^{\prime}}{f(s z)-f(t z)}\right)\right|<p\left(\frac{1-\alpha}{\alpha}\right),
$$

then $f(z) \in \mathcal{N}_{\alpha}^{p} \mathcal{S}(s, t)$, where $\frac{p}{p+1}<\alpha<1$.

Proof. Let

$$
q(z)=\frac{p(f(s z)-f(t z))}{\left(s^{p}-t^{p}\right) z f^{\prime}(z)}
$$

Then $q(z)$ is clearly analytic in $\mathbb{E}$ such that $q(0)=1$. After logarithmic differentiation and some simple computation, we have

$$
z\left(\frac{1}{q(z)}\right)^{\prime} q(z)=1+\frac{z f^{\prime \prime}(z)}{f^{\prime}(z)}-\frac{z(f(s z)-f(t z))^{\prime}}{f(s z)-f(t z)} .
$$

From (16) and (17), we find that

$$
z\left(\frac{1}{q(z)}\right)^{\prime}=\frac{\left(s^{p}-t^{p}\right) z f^{\prime}(z)}{p(f(s z)-f(t z))}\left(1+\frac{z f^{\prime \prime}(z)}{f^{\prime}(z)}-\frac{z(f(s z)-f(t z))^{\prime}}{f(s z)-f(t z)}\right) .
$$

Now by condition (15), we have

$$
z\left(\frac{1}{q(z)}\right)^{\prime} \prec p\left(\frac{1-\alpha}{\alpha}\right) z=\Theta(z),
$$

where $\Theta(0)=0$. Applying Lemma 3, we have

$$
\frac{1}{q(z)} \prec 1+\int_{0}^{z} \frac{\Theta(t)}{t} d t=\frac{\alpha+p(1-\alpha) z}{\alpha},
$$

which implies that

$$
q(z) \prec \frac{\alpha}{\alpha+p(1-\alpha) z}=H(z) .
$$


We can write

$$
\begin{aligned}
\operatorname{Re}\left(1+\frac{z H^{\prime \prime}(z)}{H^{\prime}(z)}\right) & =\operatorname{Re}\left(\frac{\alpha-p(1-\alpha) z}{\alpha+p(1-\alpha) z}\right) \\
& \geq \frac{\alpha-p(1-\alpha)}{\alpha+p(1-\alpha)} .
\end{aligned}
$$

Now since $\frac{p}{1+p}<\alpha<1$, therefore we have

$$
\operatorname{Re}\left(1+\frac{z H^{\prime \prime}(z)}{H^{\prime}(z)}\right)>0
$$

This shows that $H$ is convex univalent in $\mathbb{E}$ and $H(\mathbb{E})$ is symmetric about the real axis, therefore

$$
\operatorname{Re}(H(z)) \geq H(1) \geq 0 .
$$

Combining (16), (18), and (19), we deduce that

$$
\operatorname{Re}(q(z))>\alpha,
$$

which implies that $f(z) \in \mathcal{N}_{\alpha}^{p} \mathcal{S}(s, t)$.

Author Contributions: Conceptualization, S.M., M.A. and H.M.S.; methodology, S.M. and M.A.; software, E.S.A.A.; validation, S.M., M.A. and H.M.S.; formal analysis, S.M.; investigation, S.M.; resources, F.G.; data curation, S.M. and M.A.; writing-original draft preparation, S.M.; writing-review and editing, E.S.A.A.; visualization, S.M. and H.M.S.; supervision, S.M. and M.A.; project administration, S.M.

Funding: This research received no external funding.

Acknowledgments: The authors would like to thank the reviewers of this paper for his/her valuable comments on the earlier version of the paper. They would also like to acknowledge Salim ur Rehman, Sarhad University of Science \& Information Technology, for providing excellent research and academic environment.

Conflicts of Interest: The authors declare no conflict of interest.

\section{References}

1. Miller, S.S.; Mocanu, P.T. Differential subordinations and inequalities in the complex plane. J. Differ. Equ. 1987, 67, 199-211. [CrossRef]

2. Uralegaddi, B.A.; Ganigi, M.D.; Sarangi, S.M. Univalent functions with positive coefficients. Tamkang J. Math. 1994, 25, 225-230.

3. Owa, S.; Nishiwaki, J. Coefficient estimates for certain classes of analytic functions. J. Ineq. Pure Appl. Math. 2002, 3, 72.

4. Nunokawa, M.; Owa, S.; Polattoglu, Y.; Caglar, M.; Duman, E.Y. Some sufficient conditions for starlikeness and convexity. Turk. J. Math. 2010, 34, 333-337.

5. Owa, S.; Srivastava, H.M. Some generalized convolution properties associated with certain subclasses of analytic functions. J. Ineq. Pure Appl. Math. 2002, 3, 42.

6. Polatoğlu, Y.; Blocal, M.; Sen, A.; Yavuz, E. An investigation on a subclass of $p$-valently starlike functions in the unit disc. Turk. J. Math. 2007, 31, 221-228.

7. Dixit, K.K.; Pathak, A.L. A new class of analytic functions with positive coefficients. Ind. J. Pure. Appl. Math. 2003, 34, 209-218.

8. Uyanik, N.; Shiraishi, H.; Owa, S.; Polatoğlu, Y. Reciprocal classes of p-valently spirallike and p-valently Robertson functions. J. Ineq. Appl. 2011, 2011, 61. [CrossRef]

9. Arif, M.; Umar, S.; Mahmood, S.; Sokol, J. New reciprocal class of analytic functions associated with linear operator. Iran. J. Sci. Technol. Trans. A Sci. 2018, 42, 881. [CrossRef]

10. Arif, M. Sufficiency criteria for a class of $p$-valent analytic functions of complex order. Abstr. Appl. Anal. 2013, 2013, 517296. [CrossRef] 
11. Ponnusamy, S.; Singh, V. Criteria for strongly starlike functions. Complex Var. Theory Appl. 1997, 34, $267-291$. [CrossRef]

12. Ravichandran, V.; Selvaraj, C.; Rajalakshami, R. Sufficient conditions for starlike functions of order $\alpha$. J. Ineq. Pure Appl. Math. 2002, 3, 81.

13. Sokół, J.; Spelina, L.T. On a sufficient condition for strongly starlikeness. J. Ineq. Appl. 2013, $2013,383$. [CrossRef]

14. Uyanik, N.; Aydogan, M.; Owa, S. Extension of sufficient conditions for starlikeness and convexity of order a. Appl. Math. Lett. 2011, 24, 1393-1399. [CrossRef]

15. Yang, D.-G. Some criteria for multivalently starlikeness. Southeast Asian Bull. Math. 2000, 24, 491-497. [CrossRef]

16. Arif, M.; Ayaz, M.; Aouf, M.K. New criteria for functions to be in a class of $p$-valent alpha convex functions. Sci. World J. 2013, 2013, 280191. [CrossRef] [PubMed]

17. Arif, M.; Ayaz. M.; Iqbal, J.; Haq, W. Sufficient conditions for functions to be in a class of $p$-valent analytic functions. J. Comput. Anal. Appl. 2013, 16, 159-164.

18. Jack, I.S. Functions starlike and convex of order $\alpha$. J. Lond. Math. Soc. 1971, 3, 469-474. [CrossRef]

(c) 2019 by the authors. Licensee MDPI, Basel, Switzerland. This article is an open access article distributed under the terms and conditions of the Creative Commons Attribution (CC BY) license (http://creativecommons.org/licenses/by/4.0/). 